\title{
Research Paper \\ A Comparison of the Effect of Massage With Lavender Gel and Piroxicam Gel on Exercise-induced Muscle Soreness in Male Students of Gonabad University of Medical Sciences
}

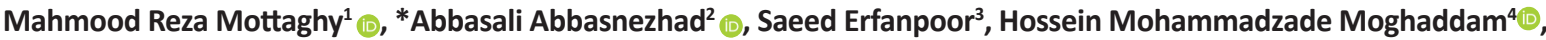 \\ Mohammad Reza Arbaghaei ${ }^{5}$, Zahra Rouhani ${ }^{1}$
}

\author{
1. Department of General Fields, School of Medicine, Gonabad University of Medical Sciences, Gonabad, Iran \\ 2. Department of Physiology, School of Medicine, Gonabad University of Medical Sciences, Gonabad, Iran \\ 3. Department of Epidemiology, School of Public Health, Iran University of Medical Sciences, Tehran, Iran. \\ 4. Department of Community Medicine, School of Medicine, Gonabad University of Medical Sciences, Gonabad, Iran. \\ 5. Department of Internal, School of Medicine, Gonabad University of Medical Sciences, Gonabad, Iran.
}

\begin{tabular}{|l|l|l}
\hline $\begin{array}{c}\text { Use your device to scan } \\
\text { and read the article online }\end{array}$ & $\begin{array}{l}\text { Citeation Mottaghy MR, Abbasnezhad A, Erfanpoor S, Mohammadzade Moghaddam H, Arbaghaei MR, Rouhani Z. [A Com- } \\
\text { parison of the Effect of Massage With Lavender Gel and Piroxicam Gel on Exercise-induced Muscle Soreness in Male Students of } \\
\text { Gonabad University of Medical Sciences (Persian)]. Quarterly of "The Horizon of Medical Sciences". 2020; 26(3):200-211. https:// } \\
\text { doi.org/10.32598/hms.26.3.1871.7 }\end{array}$ \\
dol https://doi.org/10.32598/hms.26.3.1871.7
\end{tabular}

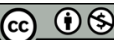

Received: 23 Jul 2019

Accepted: 29 Dec 2019

Available Online: 01 Jul 2020

Key words:

Lavender, Piroxicam,

Myalgia, Massage

\section{A B STRACT}

Aims Muscular pain is a common complication of physical activity. The purpose of this study was to compare the effect of massage with lavender gel and piroxicam gel on exercise-induced muscle pain in male students of Gonabad University of Medical Sciences.

Methods \& Materials This semi-experimental study was performed on 75 male students of Gonabad University of Medical Sciences in 2018. The Samples were randomly divided into three groups of 25, including a lavender gel massage group, a piroxicam gel massage group, and a placebo gel massage group. The specimens were randomly divided into three groups of 25 , including a lavender gel massage group, a piroxicam gel massage group, and a placebo gel massage group. The training program lasted for 45 minutes, including 10 minutes of warming up and doing stretching exercises, 30 minutes of exercises and the main exercises and 5 minutes for cooling. The research instrument was a checklist for the demographic characteristics of the samples and the Thalog malformation questionnaire. Samples completed the Thalog pain questionnaire after completing the exercise and also after the massage. The Mean \pm SD were used for descriptive statistics. Normaly distributed data were analyticaly analyzed by paired t-test and ANOVA and non-normaly distributed data were analyticaly analyzed by Wilcoxon and Kruskal-Wallis tests.

Findings The results showed that muscle pain induced by exercise in all three groups after massage was significantly reduced compared to before massage $(P<0.001)$. The results of Kruskal-Wallis test showed that the difference in muscle pain before and after massage with the three gels used

$(\mathrm{P}=0.002)$ was statistically significant. Massage with lavender gel compared to piroxicam and placebo groups was able to reduce more the muscle pain.

Conclusion Massage with lavender gel compared to the piroxicam gel and placebo gel can be a better way to reduce muscle pain after exercise.

\section{* Corresponding Author:}

Abbasali Abbasnezhad, PhD.

Address: Department of Physiology, School of Medicine, Gonabad University of Medical Sciences, Gonabad, Iran.

Tel: +98 (51) 57225027

E-mail: abbasnezhad.abbasali@gmail.com 


\section{Extended Abstract}

\section{Introduction}

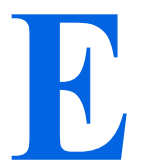

xercise reduces several physical and psychological disorders, such as anxiety and depression, and increases self-confidence and self-esteem [1]. Considering the intensity, sports activities are divided into two types of aerobic and anaerobic. Aerobic exercise is performed with an intensity of less than $70 \%$ of the maximum heart rate. It usually takes more than 2 min of continuous activity, which is done both continuously and periodically. Fat burning and weight loss are the most important features of aerobic exercise. However, periodic physical activities performing with the intensity of more than $70 \%$ of a person's maximum heart rate through less than $2 \mathrm{~min}$ are effective in reducing lactic acid accumulation and a delay the fatigue [2].

Muscle soreness (pain) is one of the most common sports injuries that occur frequently in people with different levels of physical fitness and is divided into the acute and delayed soreness depending on its severity and causes [3]. Acute muscle soreness is temporary and usually occurs a few minutes to a few hours after strenuous activity. The main cause of acute muscle soreness is ischemia, leading to the accumulation of metabolic products (lactic acid and potassium) [4]. Delayed soreness occurs about 8-12 h after strenuous exercise and reaches its maximum level after 24-48 h, followed by a gradual decrease and disappearing completely after 5-7 days. Following prolonged vigorous exercise, damage to muscle cell membranes triggers inflammatory responses and the release of the enzymes, such as Lactate Dehydrogenase (LDH), Creatine Kinase (CK), and aspartate aminotransferase [3].

Massage therapy is one of the treatments for muscle soreness. Massage is a series of regular and principled movements that are given to body tissues for specific purposes. Pain relief is one of the important usages of massage because, on the one hand, it increases blood flow and removes waste products from the tissues, and on the other hand, increases the pain threshold of neuroreceptors and reduces the sensitivity of pain receptors [5]. In addition to massage therapy, stretch therapy, cold therapy, and Non-Steroidal Anti-Inflammatory Drugs (NSAIDs) are also used to reduce muscle soreness caused by exercise [6]. Piroxicam is one of the most effective nonsteroidal anti-inflammatory painkillers commonly used to treat inflammation and pain due to rheumatoid arthritis, osteoarthritis, and acute muscle soreness. Piroxicam topical gel is also commonly used for local inflammation and pain [7].
The use of medicinal plants has increased in recent years due to their lower side effects and several effective compounds. Lavender (lavandula angustifolia) [8] is a perennial and evergreen plant of Lamiaceae (the mint family) with blue, purple, and very fragrant flowers that has been used in traditional medicine. It has more than 40 different compounds, of which linalool, linalyl acetate, cineole, nerol, borneol, butyric acid, propionic acid, hydrochloric acid, free linalool, geraniol, tannins, and flavonoids are the most important $[9,10]$. The effectiveness of Lavender on the body organs and cells as well as its analgesic and antiinflammatory effects have been reported [11-13].

The analgesic effects of lavender due to its linalool content have been evaluated and confirmed in several studies [14]. Back massage with lavender essential oil as one of the non-pharmacological methods with side effects on mother and fetus shortened the duration of the first and second stages of labor and the severity of active labor pain [15]. Lavender has antioxidant [13], anti-anxiety [16], anti-epileptic [17], antidepressant [18], anticancer [21] and antifungal [23] effects. It is also used to treat insomnia [19], reduce morphine tolerance and dependence on morphine [20], and regulate the immune system [22]. This study aimed at comparing the effect of massage with lavender gel and piroxicam gel on muscle soreness caused by exercise in male students of the Gonabad University of Medical Sciences.

\section{Materials and Methods}

In this semi-experimental study, the needed data for measuring the dependent variables were collected by the pre-test and post-test method, and the effect of independent variables on dependent variables in experimental subjects was investigated. The used sampling method was convenience sampling. First, the male students of Gonabad University of Medical Sciences, who had chosen the "General Physical Education 1" course in the first semester of the academic year of 2018-19 who met the inclusion criteria, were selected as the sample and signed the informed consent. The inclusion criteria were personal and informed consent to participate in the study, physical health, no history of smoking, no allergies and underlying diseases, no drug use, and no history of professional sports.

Then, the subjects were randomly divided into three groups of 25 cases, including a group receiving the massage with placebo (lubricant gel), a group receiving the massage with lavender (topical gel, 60 g, Kimia Shafa Pharmaceutical Company) and a group receiving the massage with piroxicam (topical gel, 60 g, 0.5\%, Razak Pharmaceutical Company). The subjects randomly selected one of the three balls marked 1,2, and 3 and were placed in the group 
corresponding to that number (No. 1: The lavender massage group; No. 2: The piroxicam massage group; and No.: 3 The placebo massage group). The research samples participated in a training program designed by a physical education instructor in three different days of the week and answered the Muscle Soreness Questionnaire (MSQ) before and after the massage. The training program consisted of 10-15 min for warm-up (stretching exercises), 30-40 min for main activity (strength and endurance exercises), and $10 \mathrm{~min}$ for cooling (stretching exercises and light stretches).

The subjects in all three groups using the massage technique reduced their muscle soreness for 10-20 min under the supervision of the masseur (before starting the study, the subjects were informed how to perform the massage correctly). In this study, classical massage was performed on large muscles of the body, especially muscles of the lower torso, including the quadriceps femoris muscle, calf, gastrocnemius muscle, and hamstrings, because there were more pain and fatigue in these muscles. In this massage, up and down, circular, and tapping techniques were applied.

After the exercise, each group rubbed 2-3 g (a thin layer) of their exclusive gel on the target area and began massaging under the supervision of the masseur. At the end of the massage, the subjects reanswered the MSQ. This questionnaire is scored from 0-6 (0: Complete absence of pain, 1: Vague and unknown feeling of pain, 2: Light and continuous pain, 3: more pain than before, 4: Excruciating pain, 5: Severe pain, and 6: unbearable pain that might result from bruising of muscle damage). The validity and reliability of the questionnaire confirmed in Arefinia study (?).

\section{Statistical analysis}

In this study, SPSS software V. 22 was used to analyze the data and the results were analyzed using descriptive and analytical statistical methods. After checking the accuracy of the data, central (Mean) and dispersion (Standard Deviation) measures were used for quantitative variables. For describing qualitative variables, "frequency" and "frequency percentage" were used. In the analytical analysis, Analysis of Variance (ANOVA) was used to compare weight, height, and Body Mass Index (BMI) variables in three groups of lavender, piroxicam, and placebo due to the normal distribution of variables. Regarding age, due to non-normally distributed data, the Kruskal-Wallis test was used. The Wilcoxon test was applied to compare the pain variables before and after the intervention in the groups. The Kruskal-Wallis test was employed to compare the effect of massage with lavender gel, piroxicam gel, and placebo gel on muscle soreness. All tests considered $\mathrm{P}<0.05$ as the significance level.

\section{Results}

In this study, 75 male students of Gonabad University of Medical Sciences participated. The Mean \pm SD of age, height, weight, and BMI of the participants were $19.36 \pm 0.96,175.20 \pm 6.58,72.99 \pm 11.40$, and $23.78 \pm 3.45$, respectively. The results showed that height $(\mathrm{P}=0.296)$, weight $(\mathrm{P}=0.791)$, BMI $(\mathrm{P}=0.840)$, and age $(\mathrm{P}=0.261)$ showed no statistically significant difference among the groups.

Muscle soreness after massage with lavender gel was significantly lower than before massage $(\mathrm{P}<0.001)$. Also, muscle soreness after massage with piroxicam gel was significantly lower than before massage $(\mathrm{P}<0.001)$. In the placebo group (ECG gel), the muscle soreness after massage with placebo significantly decreased compared with before

Table 1. Comparison of the mean soreness score based on the Muscle Soreness Questionnaire

\begin{tabular}{|c|c|c|c|c|c|c|}
\hline \multicolumn{3}{|c|}{ Soreness (Pain) } & \multirow{2}{*}{$\begin{array}{c}\text { Median } \\
3\end{array}$} & Interquartile Range & \multirow[t]{2}{*}{$\mathbf{P} *$} & \multirow[t]{2}{*}{$P^{\#}$} \\
\hline \multirow{9}{*}{ Groups } & \multirow{3}{*}{ Lavender } & Before the massage & & \multirow[t]{2}{*}{2} & & \\
\hline & & & & & \multirow[t]{2}{*}{$<0.001$} & \multirow{8}{*}{0.002} \\
\hline & & After the massage & 1 & 1 & & \\
\hline & & Before the massage & 3 & \multirow[t]{2}{*}{2} & \multirow{3}{*}{$<0.001$} & \\
\hline & Piroxicam & & & & & \\
\hline & & After the massage & 0 & 1 & & \\
\hline & \multirow{3}{*}{ Placebo } & Before the massage & 2 & 1 & \multirow{3}{*}{$<0.0901$} & \\
\hline & & & & & & \\
\hline & & After the massage & 0 & 1 & & \\
\hline coxol & & & & \multicolumn{3}{|c|}{$\begin{array}{l}\text { Quarterly of } \\
\text { The Horizon of Medical Sciences }\end{array}$} \\
\hline
\end{tabular}


the massage $(\mathrm{P}<0.001)$. Also, by subtracting the median muscle soreness after a massage from the median muscle soreness prior to massage in each group, it was found that the difference in muscle soreness in the lavender group was significantly greater than the placebo group and significantly less than the piroxicam group $(\mathrm{P}=0.002)$ (Table 1$)$.

\section{Discussion}

Muscle soreness is one of the most common sports complications that occur in intense and prolonged physical activity [3]. Exercise-induced muscle soreness results from the accumulation of acids, ions, proteins, and hormones. Also, due to the damage to the cell membrane, its activity is disturbed leading to the entry of calcium from the extracellular space to the intracellular space. This also causes the release of proteases 3 and various enzymes, such as phospholipase A2.

Phospholipase A2 releases arachidonic acid from cell membrane phospholipids, thereby increasing inflammatory mediators, such as thromboxane, prostaglandins, and leukotrienes. Prostaglandins are produced by cyclooxygenase 2 and increase the sensitivity of the afferent nerve fibers in cranial nerve III and IV to chemical and mechanical stimuli, resulting in an increase in the sensation of pain in the muscles [3].

The results of our study showed that in all three groups studied, massage reduced muscle soreness in the research samples, which is consistent with the results of other relevant studies. For example, the research on the effects of massage on Delayed Onset Muscle Soreness (DOMS) and physical performance in female college athletes. The results of this study showed that through massage, DOMS reduced and improved vertical jump in the subjects [25].

In a study that examined the effect of massage on reducing pain and anxiety during childbirth, the results showed that massage is an effective and inexpensive nursing intervention that can reduce pain and anxiety during childbirth and nursing massage is effective on labor experience [26]. Another study was conducted on the effect of pre-dressing massage on pain and anxiety in burn patients, and the results showed that pre-dressing massage reduces post-dressing pain and anxiety [27].

Comparison of three methods of aromatherapy, massage therapy, and aromatherapy massage on muscle soreness in female athletes in Isfahan city also showed that there was a significant difference between the level of muscle soreness before and after the intervention in the massage and aromatherapy massage groups, whereas no significant difference was found in the aromatherapy group [1]. In another study, massage reduced DOMS by about $30 \%$ and was effective in reducing swelling, but did not affect muscle function [28].

During the massage, the pressure is placed on the capillaries and blood vessels and this pressure helps the material and blood to return from the muscles to the heart that prevents the stopping waste products across the blood and lymph. On the other hand, as a result of the pressure on the tissues, the blood vessels dilate reflexively and the amount of blood exchanged between the tissues increases [29]. Massage also improves the elasticity of muscle fibers and helps to contract muscles. It also increases the amount of oxygen in the muscle and improves the function of food absorption in muscle fibers. In general, massage is effective in gaining the lost strength of tired muscles and restores muscles to rest [30]. It reduces pain and discomfort in the area by removing waste products. Also, when the massage movements start gently followed by a gradual increase in the intensity of the movements, the sensitivity of the pain receptors may decrease and the pain threshold can increase. Also, massage by stimulating $A B$ fibers can reduce the transmission of pain to higher areas of the spinal cord and brain [27].

The results of our study showed that the difference in muscle soreness before and after massage by three gels used was statistically significant. Bazrafkan i examined the effect of lavender on muscle soreness in athletes and concluded that lavender has a significant effect on reducing muscle soreness in female handball players, and during a match, aromatherapy and massage techniques can be used to reduce the pain of the players [31].

The analgesic effects of lavender extract due to its linalool content have been evaluated and confirmed in several studies. The analgesic effects of linalool on the spinal cord have also been reported [32]. The effectiveness of lavender fragrance has been confirmed to reduce anxiety in patients in the dental [16] and maternity wards [33]. The results of another study showed that lavender oil and polyphenolic extracts have anti-inflammatory and analgesic effects [12]. In addition, the analgesic effects of lavender essential oil on pain caused by the entry of a hemodialysis needle were investigated [34]. In acupressure, lavender oil significantly reduced low back pain [35]. Aromatherapy with lavender oil extract reduces anxiety and insomnia in patients with dementia [36]. The results of other studies also showed that this plant can be used to increase the accuracy and stress relieving and analgesic effects in the treatment of healthcare staff and patients [37].

Linalool and linalool acetate in lavender can stimulate the parasympathetic system. Therefore, this plant has sedative, 
analgesic, and anticonvulsant effects. Linalool, as the most active part of the plant, has also been observed in non-central nervous system cells and tissues, indicating its spasmolytic and sedative effects. Linalyl acetate and linalool have narcotic and sedative effects, respectively. Potassium channels play a key role in the effectiveness of lavender, and they are likely to be triggered by stimulation of muscarinic M2, opioid, or dopamine D2 receptors [38].

The inhibition of nitric oxide synthesis by lavender can be one of the main mechanisms to exert its analgesic effects [14]. However, linalool, which is a competitive antagonist of NMDA receptors, inhibits the activity of these receptors and exerts its analgesic effects [39]. In another study, the analgesic effects of linalool were inhibited by atropine, naloxone, glibenclamide, and sulpiride, but were not inhibited by pirenzepine and $\mathrm{SCH}-23390$. It can be concluded that these results are consistent with the pharmacological characteristics of the plant, the effects of cholinergic analgesia, and the blocking effect of NMDA channels [38]. The results of a study showed that linalool acetate, which is one of the main components of lavender, relaxes the muscles of the rabbit's vascular wall. It was also found that this effect was achieved by dephosphorylation of the light chain of myosin and producing nitric oxide [40].

According to the proven role of calmodulin in the process of contraction and relaxation of the single- and multi-unit smooth muscles, the effect of lavender, and more precisely, the linalool acetate in this process can be attributed to the separation of calcium from calcium-activated protein kinase and muscle relaxation. Considering the effect of lavender on tolerating the analgesic effects of morphine and reducing its dependence, it has shown that inhibiting the action of calmodulin can reduce tolerance to the analgesic effects of morphine and reinforce the possible effect of lavender to reduce tolerance to morphine analgesic effects and dependence by affecting calmodulin and related kinases [20, 41$]$.

\section{Conclusion}

In all three groups studied, there was a significant relationship between massage and pain reduction in the research samples so that the samples felt less pain after exercising and massage therapy. The results also showed that massage with lavender gel had a greater effect than piroxicam gel and placebo gel on reducing muscle soreness caused by exercise.

\section{Ethical Considerations}

\section{Compliance with ethical guidelines}

This study was approved by the Vice Chancellor for Research and Technology of Gonabad University of Medical Sciences (Ethical Code: IR.GMU.REC.1397.043).

\section{Funding}

The present paper was extracted from the MSc. thesis of Mohammad Reza Arbaghaei, Department of Community Medicine, School of Medicine, Gonabad University of Medical Sciences. Also the Research Affairs of Gonabad University of Medical Sciences supported this study.

\section{Authors' contributions}

Writing-original draft, final review: All authors; Conceptualization: Mohammad Reza Mottaghy, Abbasali Abbasnezhad; Final proof: Mohammad Reza Mottaghy; Results interpretation: Saeed Erfanpoor, Hossein Mohammadzade Moghaddam, Zahra Rouhani; Design, data gathering: Mohammad Reza Arbaghaei.

\section{Conflicts of interest}

The authors declared no conflict of interest.

\section{Acknowledgements}

The authors would like to thank Research Affairs of Gonabad University of Medical Sciences for their financial support. 


\title{
مقايسه اثر ماساز با زٔل اسطوخودوس و ثل بيروكسيكام بر درد عضلاتى ناشى از ورزش
}

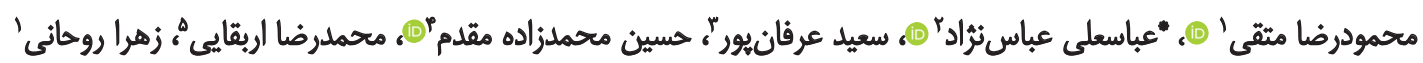

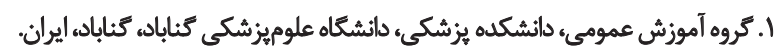

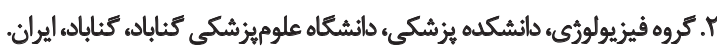

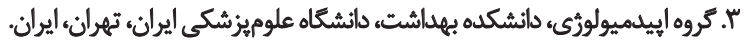

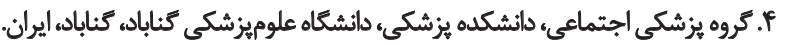

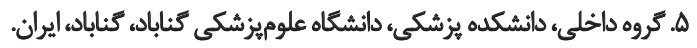

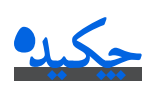

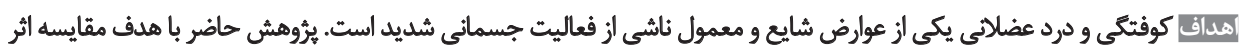

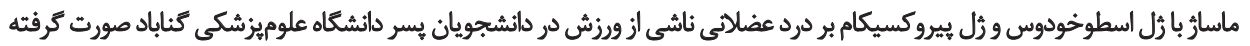

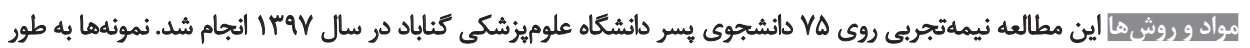

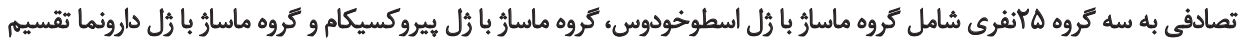

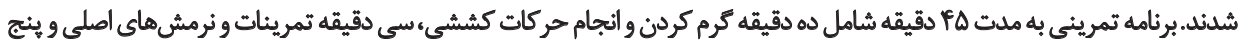

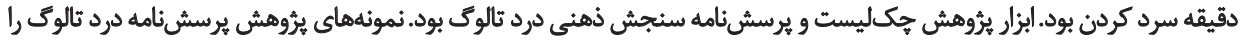

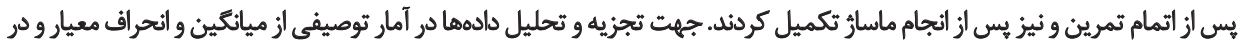

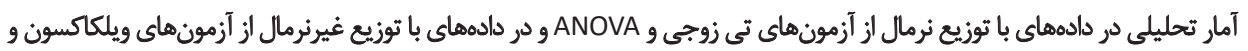

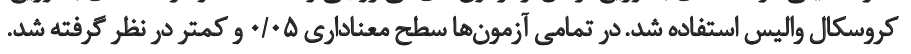

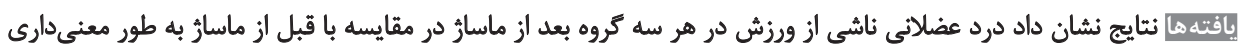

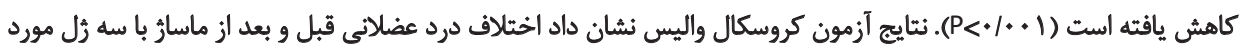

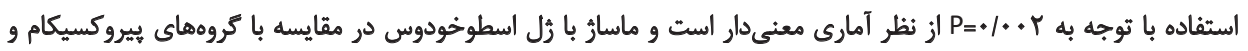

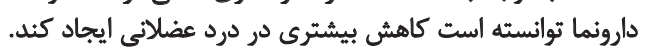

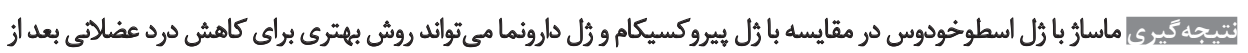

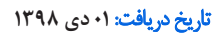

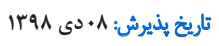
ورزش باشد.

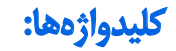

اسطوخودوس،

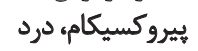
عضلاني، ماسارٌ
ضربان قلب فرد انجام شده و زمان اجراى آن كمتر از دو دقيقه

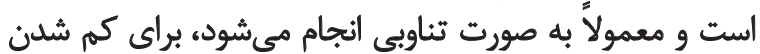

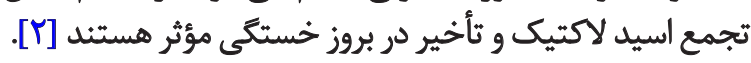
كوفتتى و درد عضلانى به عنوان يكى از رايجترين صدمات

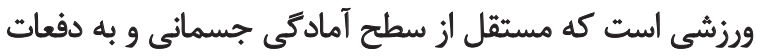

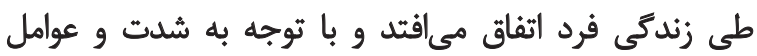

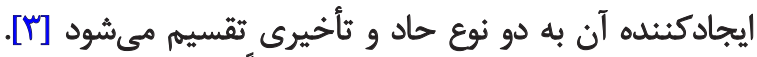

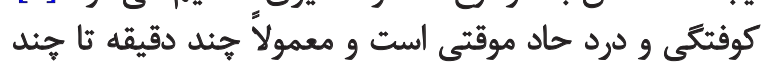

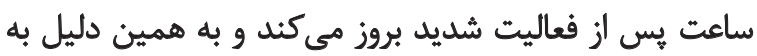

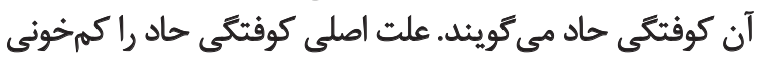

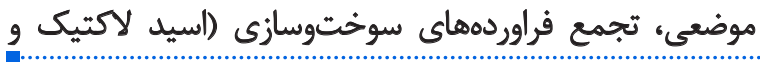

doteo

ورزش عامل مهمى در كاهش خطر ابتلا به بسيارى از

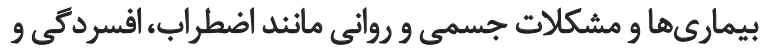

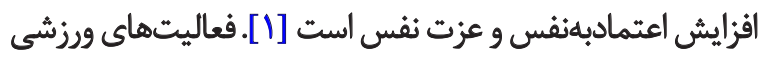

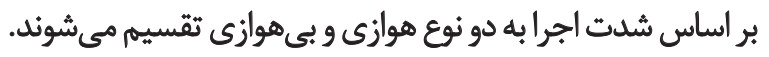

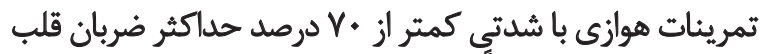

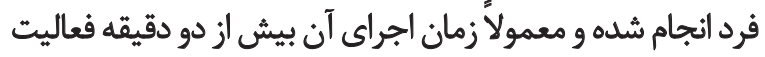

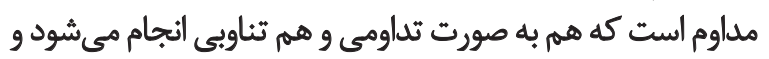

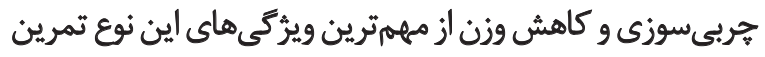

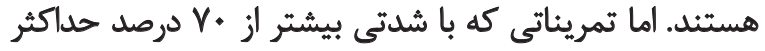

: تويسئده مسئول:

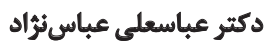

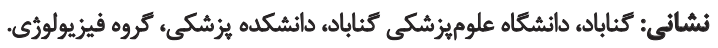

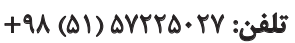

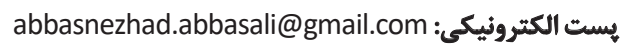




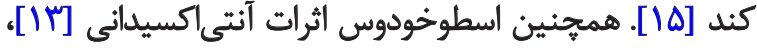

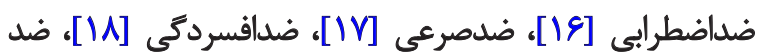

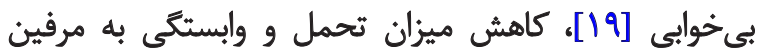

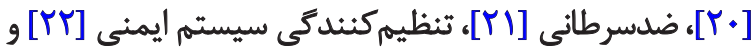

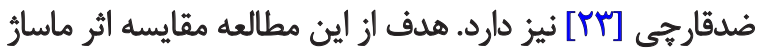

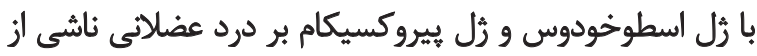

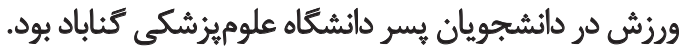

\section{مواد وروشها نها}

در اين مطالعه نيمهتجربى اطلاعات موردنظر براى اندازهكيرى

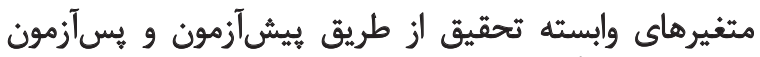

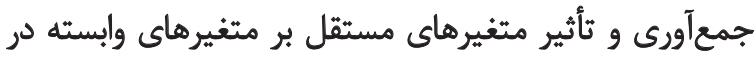

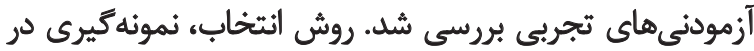

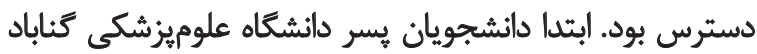

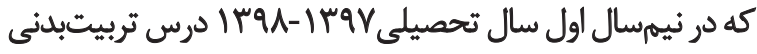

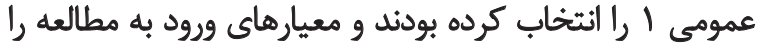

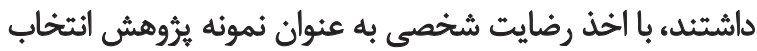

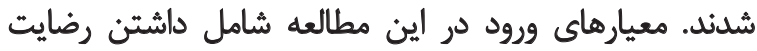

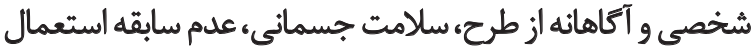

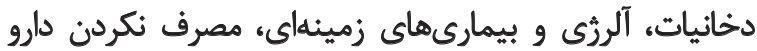

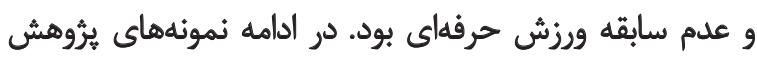

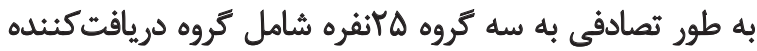

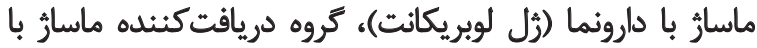

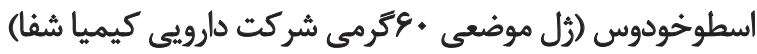

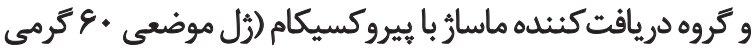

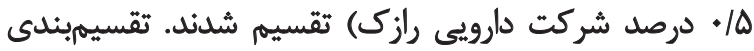

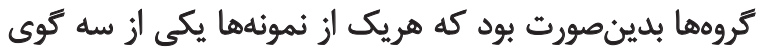

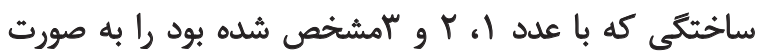

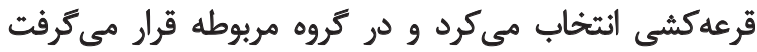

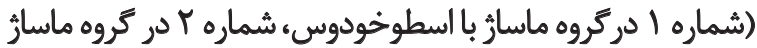

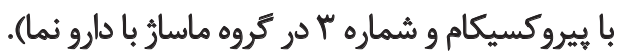

نمونههاى يُروهش در سه روز مختلف از ايام هفته در برنامه

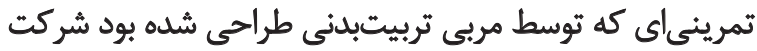

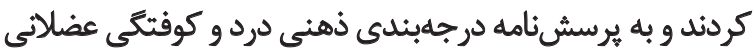

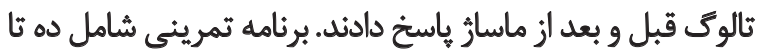

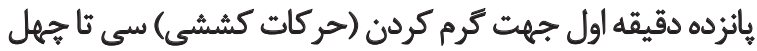

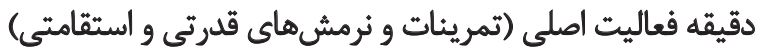

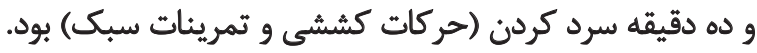

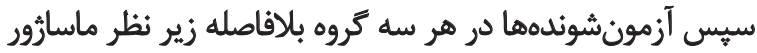

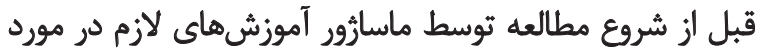

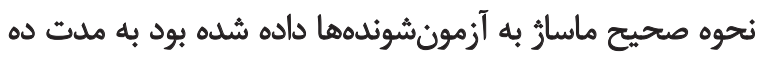

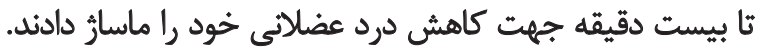

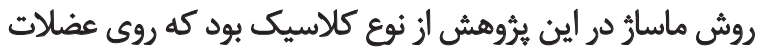

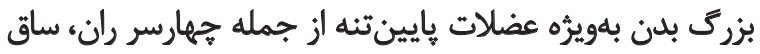

يتاسيم) دانستهاند [?[]. درد تأخيرى حدود هشت تادوازده ساعت

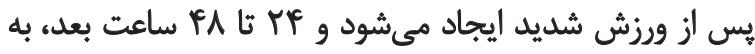

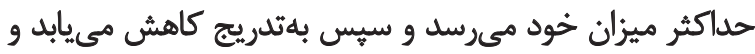

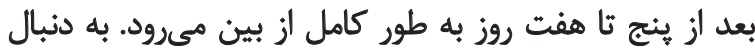

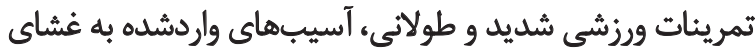

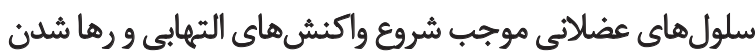

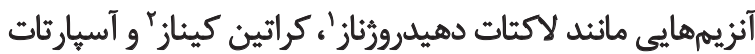

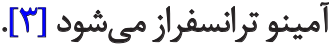

ماكى از روش هاى درمان كوفتكّى و درد عضلاتى، ماسارٌ است.

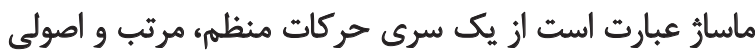

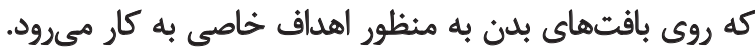

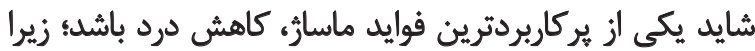

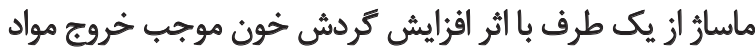

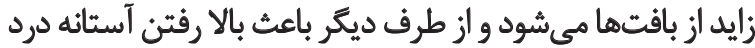

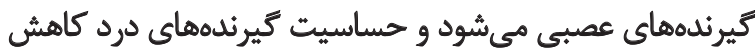

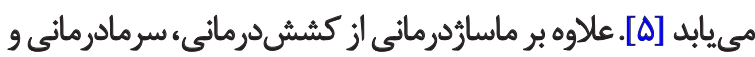

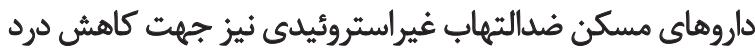
عضلاثى ناشى از ورزش استفاده مي شود [9].

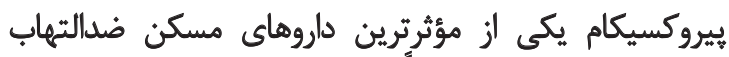

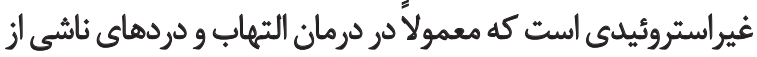

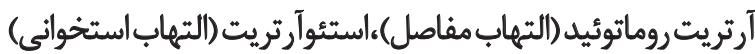

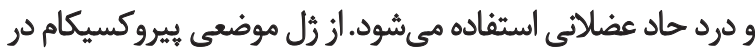

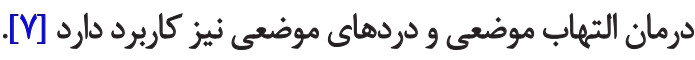

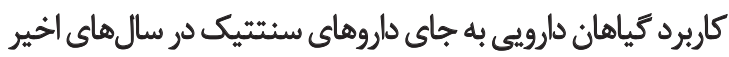
به دليل عوارض جانبي اندك و تنوع تركيبات مؤثر افزايش يافته است داست اسطوخودوس يكى از كياهانى است كه در طب سنب سنتى مورد

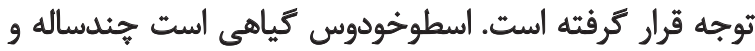

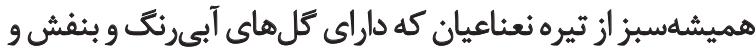
بسيار معطر است.نام علمى اسطوخودوس - Lavandula angusti

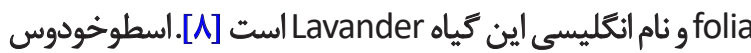

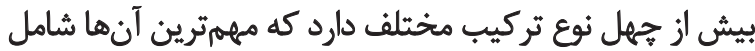

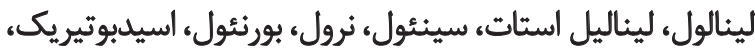

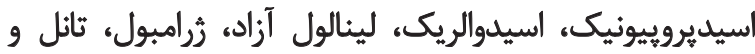

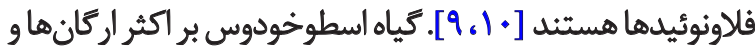

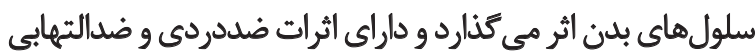

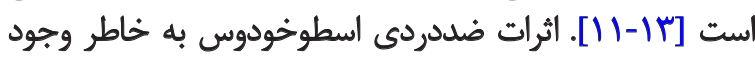

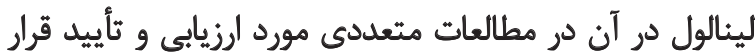

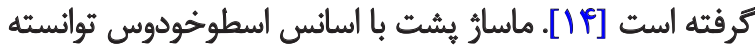

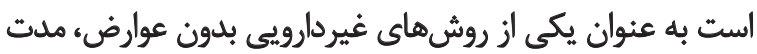

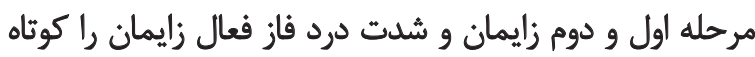

1. Lactate Dehydrogenase (LDH)

2. Creatine Kinase (CK) 


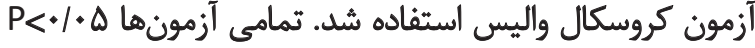
عنوان سطح معنى دارى در نظر كرفته شد.

يافتهها

در اين مطالعه VD نفر از دانشجويان يسر دانشكاه علوميزشكى مئى

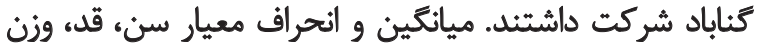

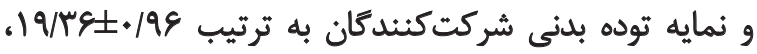
جميت

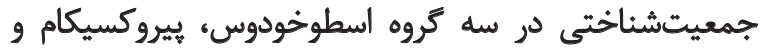

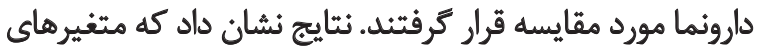

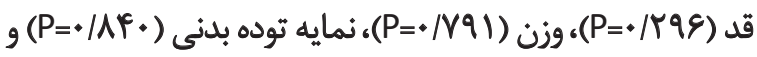

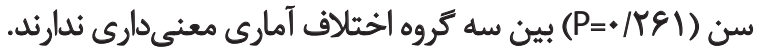

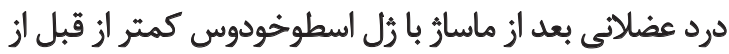

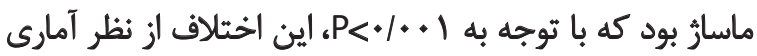

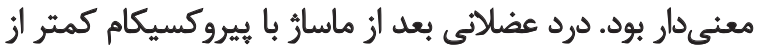

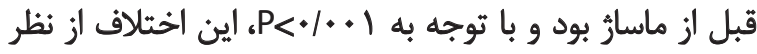

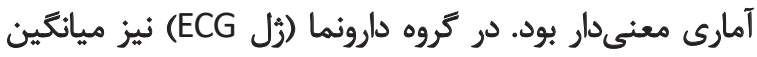

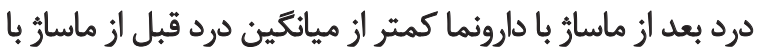

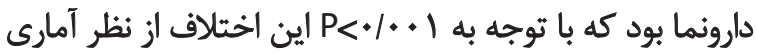

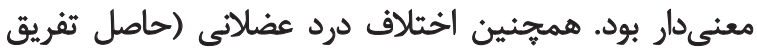

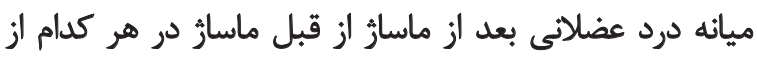

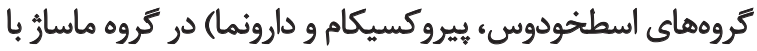

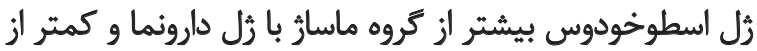

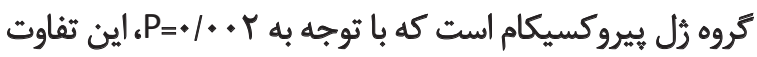

$$
\text { از نظر آمارى معنى دار است (جدول شماره بـ بام ). }
$$

\section{بحث}

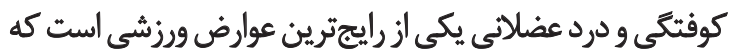

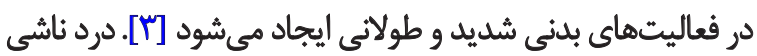

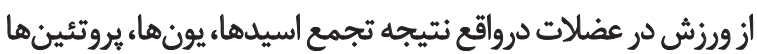

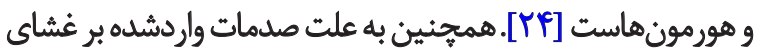

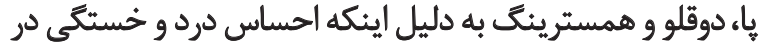

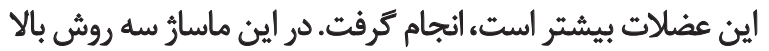

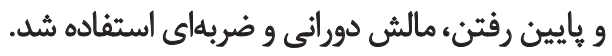

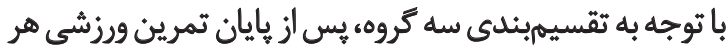

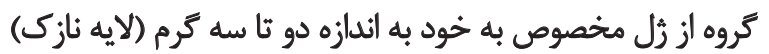

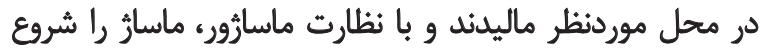

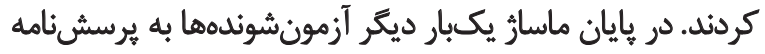

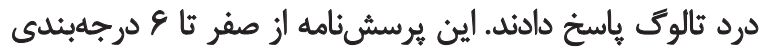

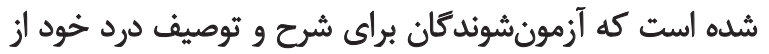

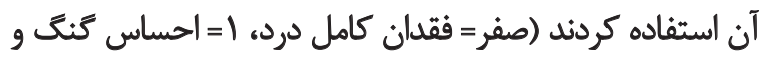

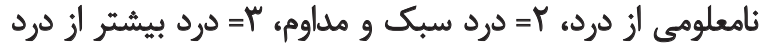

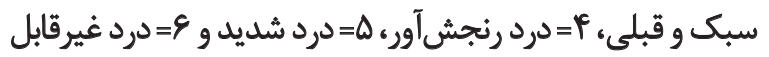

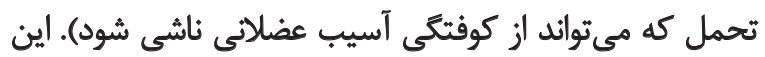

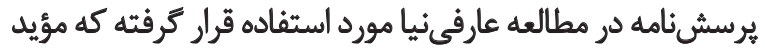

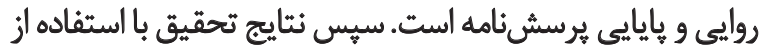
روشهاى آمارى توصيفى و تحليلى تجزيه و تحليل شدند.

\section{تحاليل آمارى}

در اين مطالعه براى آناليز دادهها از نرمافزار SPSS نسخه بردي

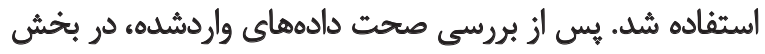

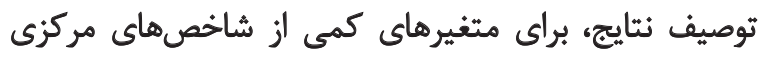

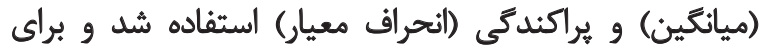

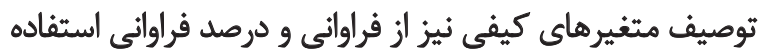

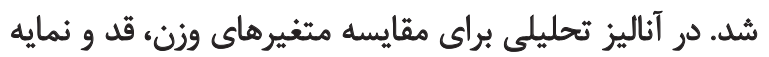

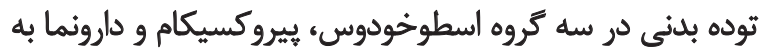

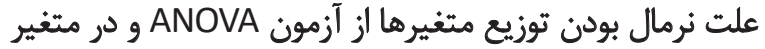

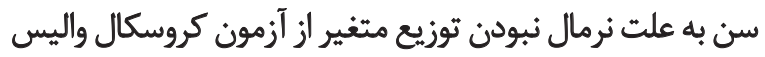

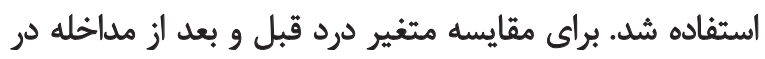

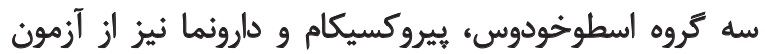

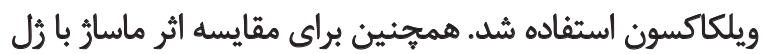
اسطوخودوس، زٔل بيروكسيكام و زل دارونما بر درد عضلانى ازئ مازئ

جدول ا. مقايسه ميانكين درد بر اساس معيار ثالوكى در سه كروه اسطوخودوس، بيروكسيكام و دارونما و مقايسه اختلاف درد عضلاتى

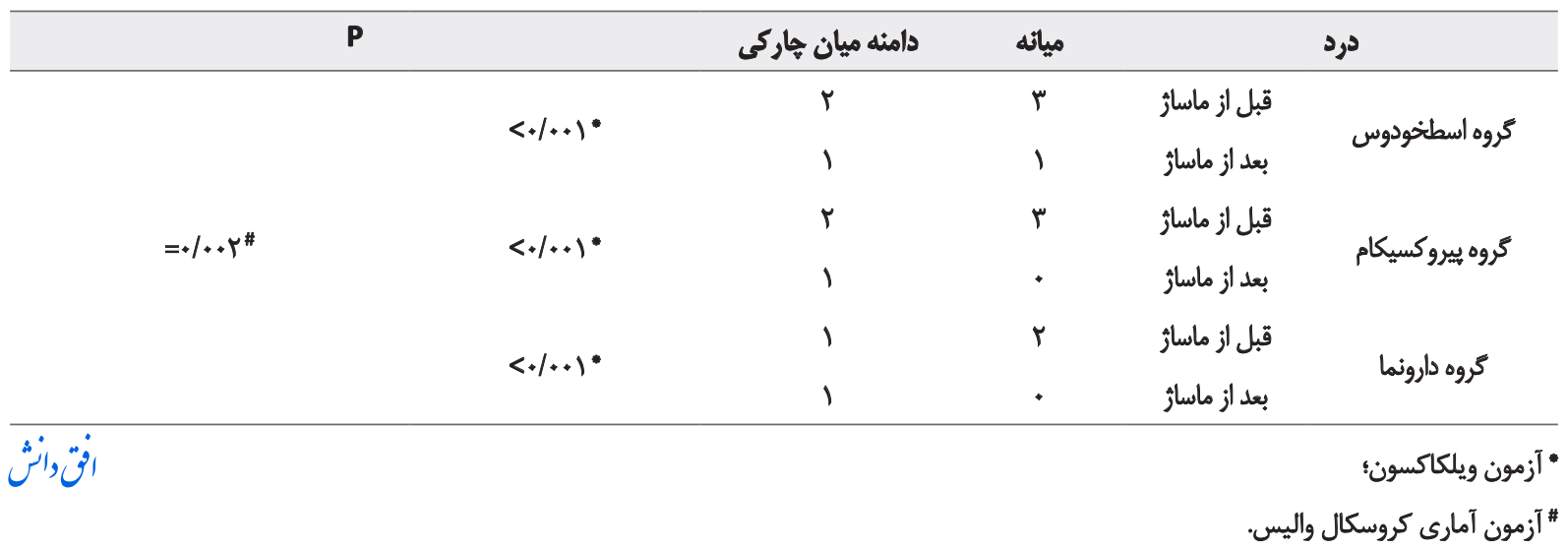


توان ازدسترفته عضلات خسته كمك مؤثرى مي كند و عضلات

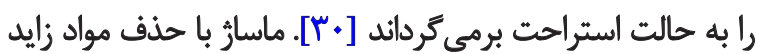

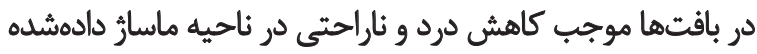

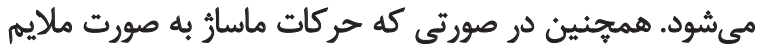

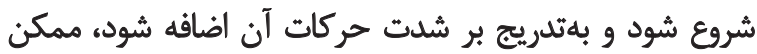

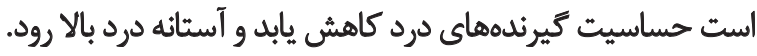

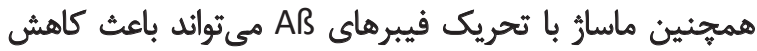

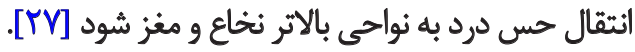
نتايج مطالعه ما نشان داد كه اختلاف درد عضلاتى قبل و بعد إندا

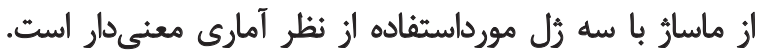

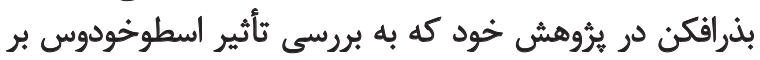

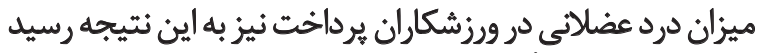

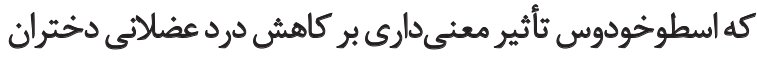

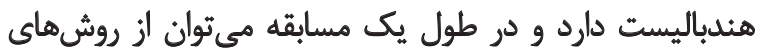

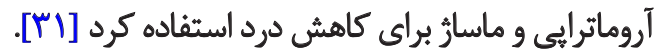

اثرات ضددردى عصاره اسطوخودوس به خاطر وجود لينالول

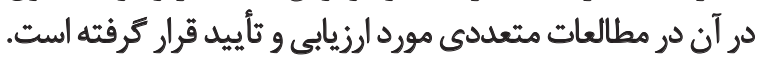

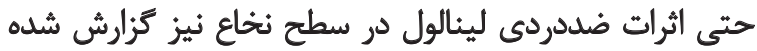

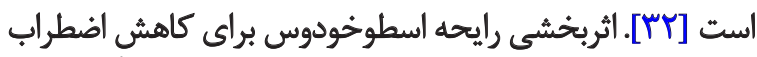

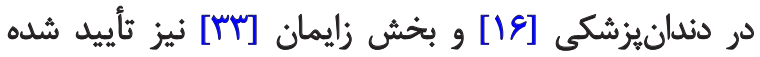

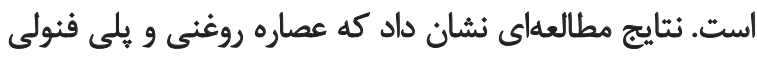

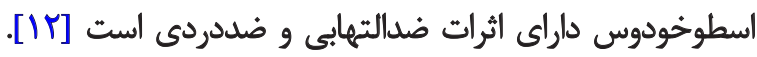

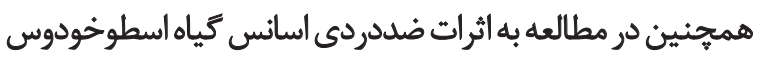

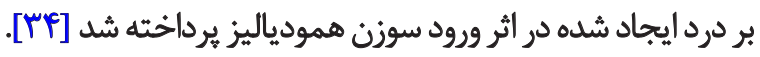

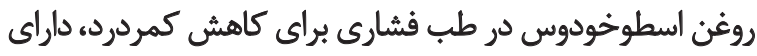

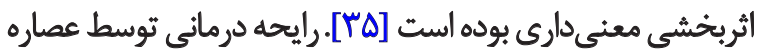

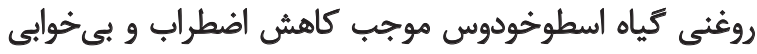

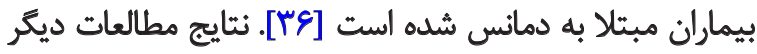

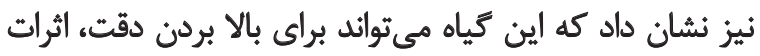

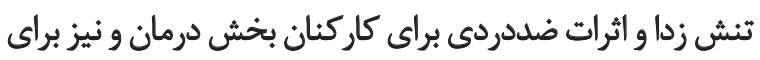

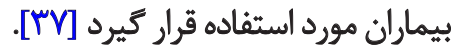

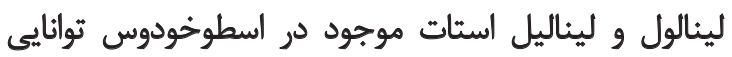

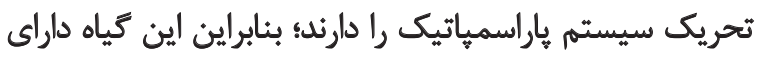

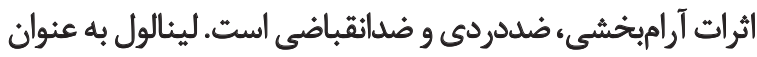

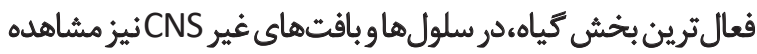

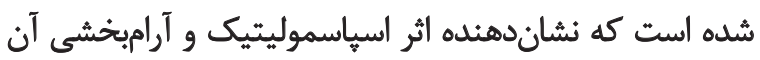

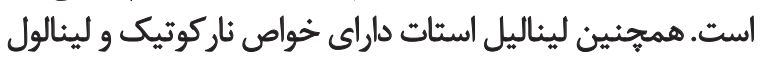

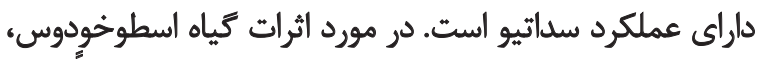

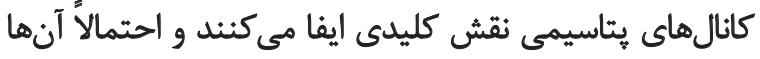

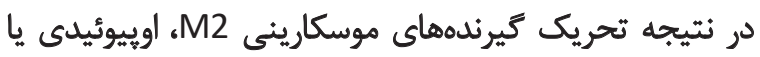

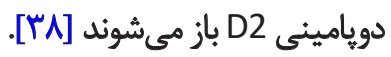

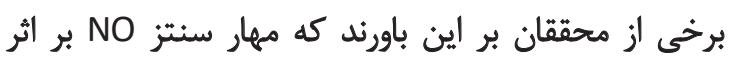

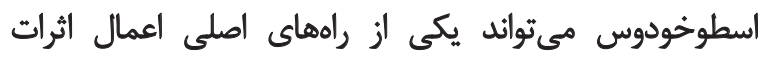

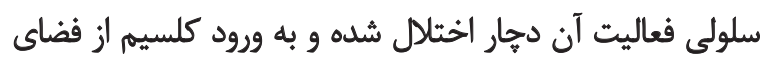

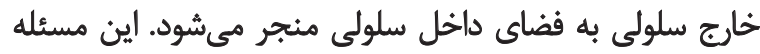

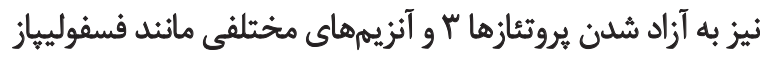

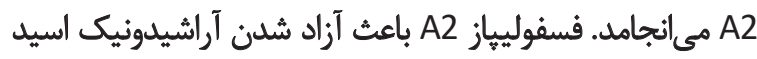

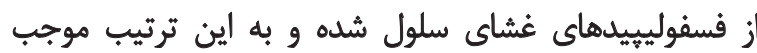

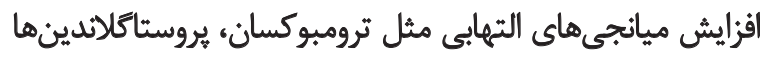

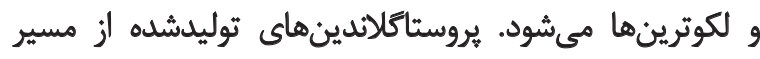

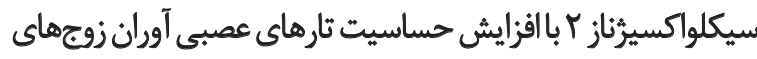

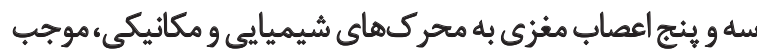

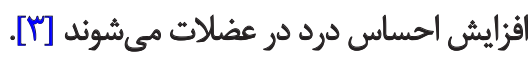

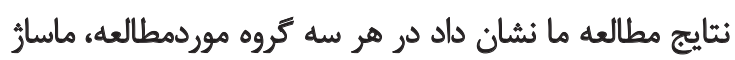

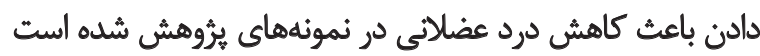

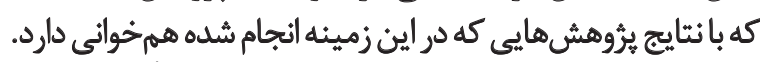
از جمله مى توان به تحقيقى اشاره كرد كه با با عنوان تأثيرات ماسراز

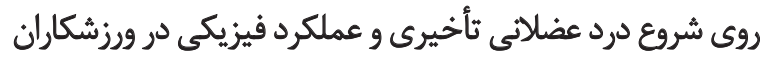

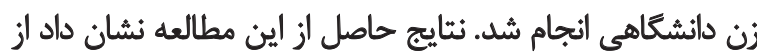

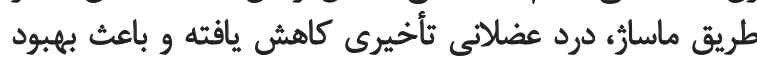

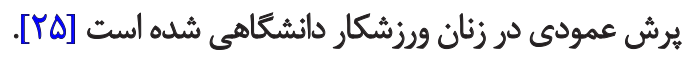

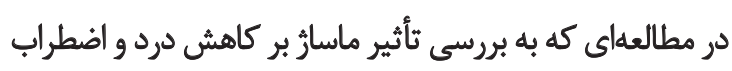

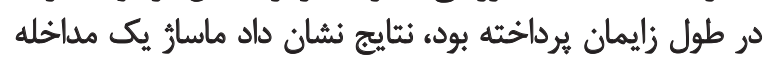

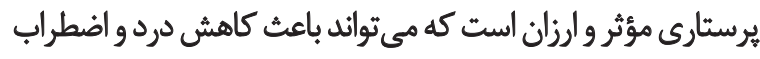

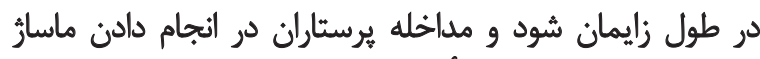

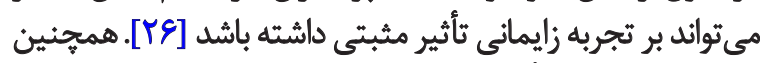

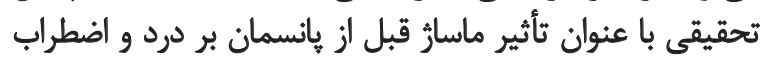

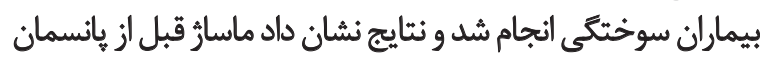

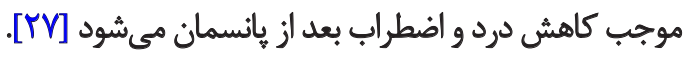

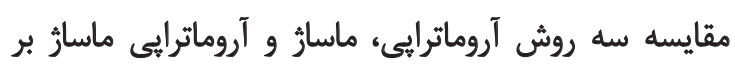

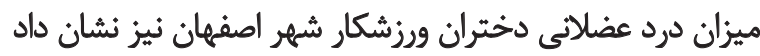

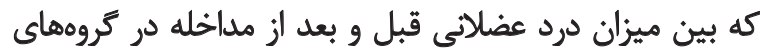

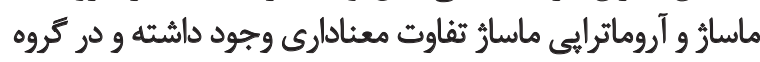

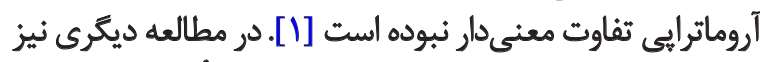

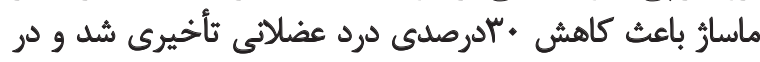

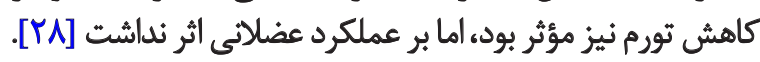

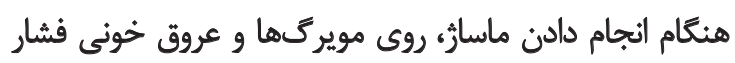

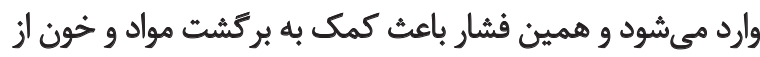

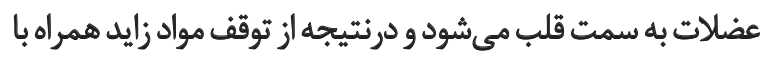

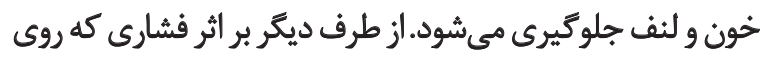

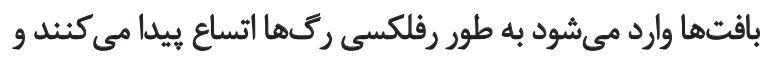

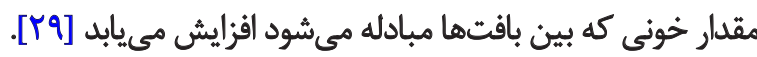

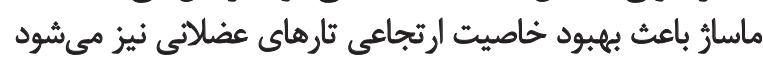

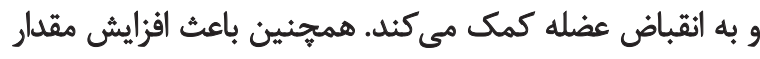

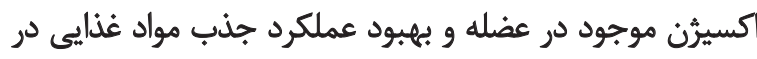

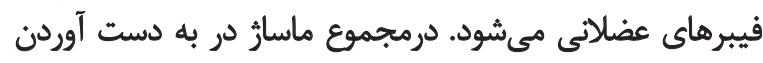


مشاركت نويسئدكان

نوشتن نسخه اوليه: همه نويسندكان؛ ايده اصلى: محمودرضيا

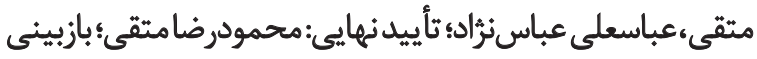

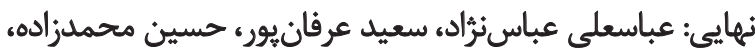

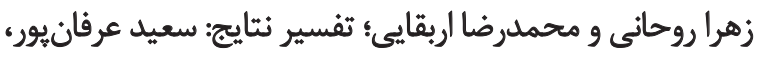

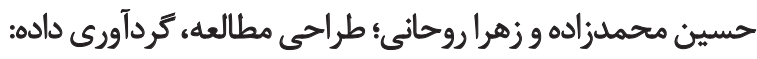

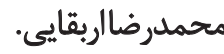

$$
\text { تعارض مثأقع }
$$

هيجّونه تعارض منافعى توسط نويسندكان بيان نشده است.

$$
\text { تشكر و قدرواني }
$$

نويسندكان بر خود لازم ميدانيد كه از معاونت تحقيقات و

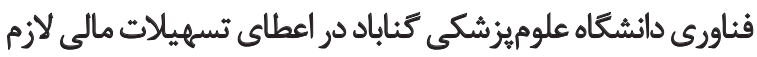
جهت أنجام اين يروزه صميمانه تشكر كنيند.
ضددردى آن باشد [If]]. با وجود اين، برخى معتقديند لينالول

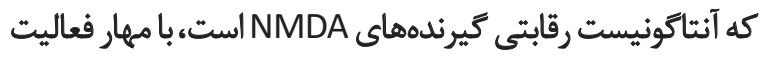

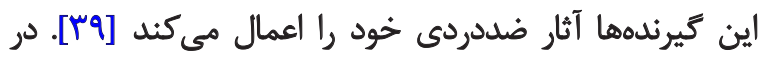

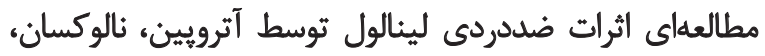

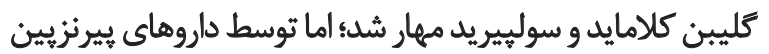

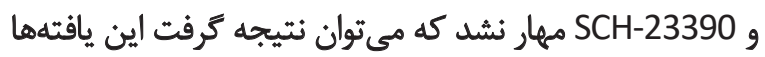

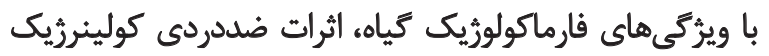

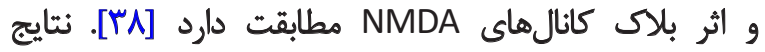

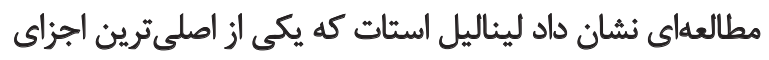

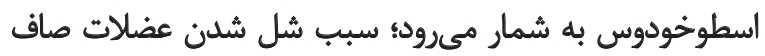

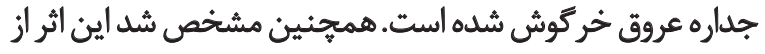

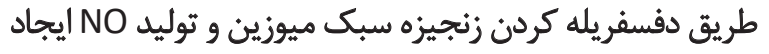

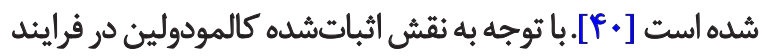

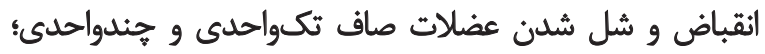

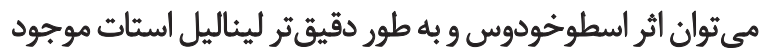

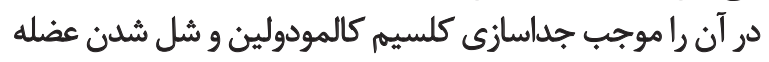

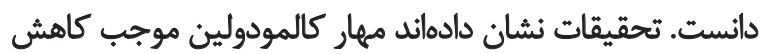

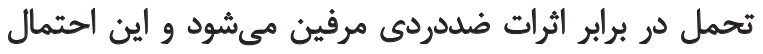

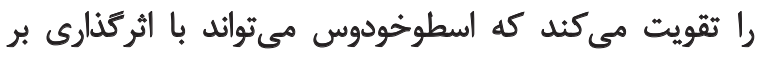

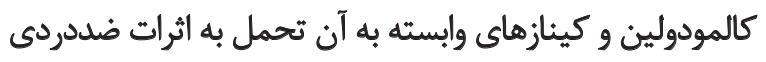

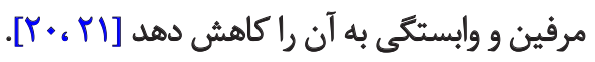

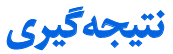

به طور خلاصه، در هر سه كروه موردمطالعه ارتباط معنادارى

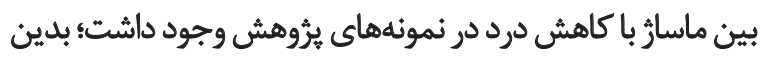

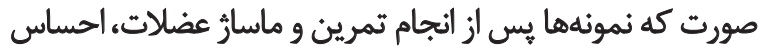

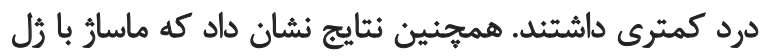

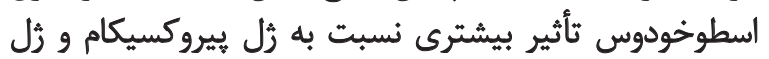
دارونما بر كاهش درد عصلانى ناشى از ورزش بارئ دارد.

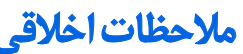

\section{يبيروى أز اصول الخلاق يُوهش}

مطابق مجوز شماره IR.GMU.REC.1397.043 معاونت

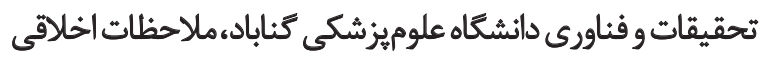
با نظارت كميته اخلاق دانشكاه علوميزشكى كثابادياد رعايت شدات

$$
\text { مامي }
$$

منابع مالى اين يروزه توسط معاونت تحقيقات و فناورى

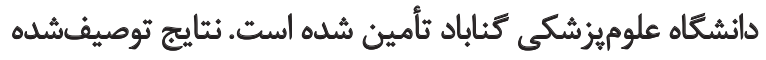

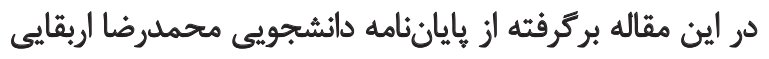

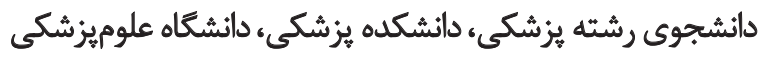

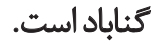




\section{References}

[1] Arefinia M, Ghasemi B, Arefinia S, Moradi MR. [The effect of three methods of aromatherapy, massage and aromatherapy-massage on muscular pain rate of female athletes in Isfahan (Iran) (Persian)]. Journal of Exercise and Science and Medicine. 2013; 5(1):55-65. https://iranjournals.nlai.ir/bitstream/handle/123456789/181423/D9134596017542B18C79DB837C013162.pdf?sequence=-1\&isAllowed=y

[2] Gamble P. Strength and conditioning for team sports: sport-specific physical preparation for high performance. Abingdon, UK: Routledge; 2013. [DOI:10.4324/9780203084250]

[3] Tartibian B, Derafshi B, Hajizadeh Maleki B, Toufighi A. [The effect of indomethacin on biochemical, functional and superficial symptoms of Delayed Onset Muscle Soreness (DOMS) in untrained men (Persian)]. Sport Biosciences (HARAKAT). 2010; 3:93-110. https://www.magiran. com/paper/763909

[4] Nejatmand N, Ramezani A, Barati AH. [Effect of consumption shortterm CoQ10 supplementation on markers of delayed onset muscle soreness (Persian)]. Razi Journal of Medical Sciences. 2014; 21(119):77-85. http://rjms.iums.ac.ir/article-1-3105-en.html

[5] Best TM, Hunter R, Wilcox A, Haq F. Effectiveness of sports massage for recovery of skeletal muscle from strenuous exercise. Clinical Journal of Sport Medicine. 2008; 18(5):446-60. [DOI:10.1097/ JSM.0b013e31818837a1] [PMID]

[6] Proske U, Morgan DL. Muscle damage from eccentric exercise: Mechanism, mechanical signs, adaptation and clinical applications. The Journal of Physiology. 2001; 537(2):333-45. [DOI:10.1111/j.14697793.2001.00333.x] [PMID] [PMCID

[7] Brogden RN, Heel RC, Speight TM, Avery GS. Piroxicam. Drugs. 1984; 28(4):292-323. [DOI:10.2165/00003495-198428040-00002] [PMID]

[8] Palá-Paúl J, John Brophy J, Goldsack RJ, Fontaniella B. Analysis of the volatile components of Lavandula canariensis (L.) Mill., a Canary Islands endemic species, growing in Australia. Biochemical Systematics and Ecology. 2004; 32(1):55-62. [DOI:10.1016/S0305-1978(03)00177-7]

[9] Morgan TJ, Morden WE, Al-Muhareb E, Herod AA, Kandiyoti R. Essential oils investigated by size exclusion chromatography and gas chromatography- mass spectrometry. Energy \& Fuels. 2006; 20(2):734-7. [DOI:10.1021/ef050364i]

[10] Barazandeh MM. Essential oil composition of Lavandula latifolia Medik from Iran. Journal of Essential Oil Research. 2002; 14(2):103-4. [D OI:10.1080/10412905.2002.9699784]

[11] Koulivand PH, Khaleghi Ghadiri M, Gorji A. Lavender and the nervous system. Evidence-based complementary and alternative medicine. 2013; 2013:681304. [DOI:10.1155/2013/681304] [PMID] [PMCID]

[12] Hajhashemi V, Ghannadi A, Sharif B. Anti-inflammatory and analgesic properties of the leaf extracts and essential oil of Lavandula angustifolia Mill. Journal of Ethnopharmacology. 2003; 89(1):67-71. [DOI:10.1016/ S0378-8741(03)00234-4]

[13] da Silva GL, Luft C, Lunardelli A, Amaral RH, da Silva Melo DA, Donadio MVF, et al. Antioxidant, analgesic and anti-inflammatory effects of lavender essential oil. Anais da Academia Brasileira de Ciências. 2015; 87(2 Suppl):1397-408. [DOI:10.1590/0001-3765201520150056] [PMID]

[14] Peana AT, D'Aquila PS, Loredana Chessa M, Moretti MDL, Serra G, Pippia P. (-)-Linalool produces antinociception in two experimental models of pain. European Journal of Pharmacology. 2003; 460(1):37-41. [DOI:10.1016/S0014-2999(02)02856-X]
[15] Mohamadkhani Shahri L, Sabet Birjandi S, Mohamadkhani Shahri H. [Effect of massage Aromatherapy with lavandula on the duration of first and second stage of labor in nulliparous women (Persian)]. Hormozgan Medical Journal. 2013; 17(2):145-54. http://eprints.hums.ac.ir/3097/1/ hmj-v17n2p145-en.pdf

[16] Lehrner J, Marwinski G, Lehr S, Johren P, Deecke L. Ambient odors of orange and lavender reduce anxiety and improve mood in a dental office. Physiology \& Behavior. 2005; 86(1-2):92-5. [DOI:10.1016/j.physs beh.2005.06.031] [PMID]

[17] Rahmati B, Khalili M, Roghani M, Ahghari P. [Anticonvulsant effect of hydro-alcoholic extract of Lavandula officinalis on seizures in pentylenetetrazol-induced kindling model in male mice (Persian)]. Daneshvar Medicine: Basic and Clinical Research Jouranl. 2012; 19(1):25-32. http:// daneshvarmed.shahed.ac.ir/article-1-528-fa.html

[18] Akhondzadeh S, Kashani L, Fotouhi A, Jarvandi S, Mobaseri M, Moin $M$, et al. Comparison of Lavandula angustifolia Mill. tincture and imipramine in the treatment of mild to moderate depression: A double-blind randomized trial. Progress in Neuro-Psychopharmacology and Biological Psychiatry. 2003; 27(1):123-7. [DOI:10.1016/S0278-5846(02)00342-1]

[19] Fismer KL, Pilkington K. Lavender and sleep: A systematic review of the evidence. European Journal of Integrative Medicine. 2012; 4(4):e436-e47. [DOI:10.1016/j.eujim.2012.08.001]

[20] Rahmati B, Baik Khormaizi A, Khalili M, Roghani M, Ansari F. [Effects of hydro-alcoholic extract of Lavandula officinalis aerial parts on acquisition and expression of morphine tolerance and dependence in male mice (Persian)]. Daneshvar Medicine: Basic and Clinical Research Jouranl. 2013; 20(1):27-38. http://daneshvarmed.shahed.ac.ir/article1-697-fa.html

[21] Yaghoobi K, Kaka GR, Davoodi S, Ashayeri H. [Therapeutic effects of Lavandula angustifolia (Persian)]. Journal of Gorgan University of Medical Sciences. 2016; 17(4):1-9. http://goums.ac.ir/journal/article1-2509-fa.pdf

[22] Azadmehr A, Hajiaghaee R, Rezazadeh S, Afshari A, Baradaran B, Ebrahimi P. [Evaluation of Lavandula officinalis extract on Lymphocyte proliferation and tumor necrosis factor-alpha production (Persian)]. Journal of Medicinal Plants. 2011; 2(38):142-7. http://jmp.ir/article1-221-fa.pdf

[23] Mahboubi M, Feizabadi MM, M. Safara. Antifungal activity of essentia oils from Zataria multiflora, Rosmarinus officinalis, Lavandula stoechas, Artemisia sieberi Besser and Pelargonium graveolens against clinical isolates of Candida albicans. Pharmacognosy Magazine. 2008. 4:15-8. https://www.researchgate.net/publication/285273440

[24] Miles MP, Clarkson PM. Exercise-induced muscle pain, soreness, and cramps. The Journal of Sports Medicine and Physical Fitness. 1994; 34(3):203-16. [PMID]

[25] Mancinelli CA, Scott Davis D, Aboulhosn L, Brady M, Eisenhofer J, Foutty $S$. The effects of massage on delayed onset muscle soreness and physical performance in female collegiate athletes. Physical Therapy in Sport. 2006; 7(1):5-13. [DOI:10.1016/j.ptsp.2005.10.004]

[26] Pilevarzadeh M, Salari S, Shafiei N. Effect of massage on reducing pain and anxiety during labour. Journal of Reproduction \& Infertility, 2002; 3(4):42-6. http://www.jri.ir/article/102

[27] Najafi Ghezeljeh T, Mohades Ardebili F, Rafii F, Manafi F. The effect of massage on anticipatory anxiety and procedural pain in patients with burn injury. World Journal of Plastic Surgery. 2017; 6(1):40-7. [PMID] [PMCID] 
[28] Zainuddin Z, Newton M, Sacco P, Nosaka K. Effects of massage on delayed-onset muscle soreness, swelling, and recovery of muscle function. Journal of Athletic Training. 2005; 40(3):174-80. [PMID] [PMCID]

[29] Weerapong P, Hume PA, Kolt GS. The mechanisms of massage and effects on performance, muscle recovery and injury prevention. Sports Medicine. 2005; 35(3):235-56. [DOI:10.2165/00007256-20053503000004] [PMID]

[30] Nadim A. [Massage therapy according to avicenna and sports massage discipline (Persian)]. Journal of Research on History of Medicine. 2015; 4:3. https://rhm.sums.ac.ir/article_42950_64fdc30c8946fea2f0ce7aa6 86d07085.pdf

[31] Bakhtshirin F, Abedi S, Yusefizoj P, Razmjooee D. The effect of aromatherapy massage with lavender oil on severity of primary dysmenorrhea in Arsanjan students. Iranian Journal of Nursing and Midwifery Research. 2015; 20(1):156-60. [PMID] [PMCID]

[32] Batista PA, de Paula Werner MF, Oliveira EC, Burgos L, Pereira P, da Silva Brum LF, et al. Evidence for the involvement of ionotropic glutamatergic receptors on the antinociceptive effect of (-)-linalool in mice. Neuroscience Letters. 2008; 440(3):299-303. [DOI:10.1016/j.neut let.2008.05.092] [PMID]

[33] Buckle J. Essential oils: Management and treatment of gynecologic infections and stressors. Sexuality, Reproduction and Menopause, 2006; 4(1):38-41. [DOI:10.1016/j.sram.2006.03.006]

[34] Bagheri-Nesami M, Espahbodi F, Nikkhah A, Shorofi SA, Charati JY. The effects of lavender aromatherapy on pain following needle insertion into a fistula in hemodialysis patients. Complementary Therapies in Clinical Practice. 2014; 20(1):1-4. [DOI:10.1016/j.ctcp.2013.11.005] [PMID]

[35] Yip YB, Tse SHM. The effectiveness of relaxation acupoint stimulation and acupressure with aromatic lavender essential oil for non-specific low back pain in Hong Kong: A randomised controlled trial. Complementary Therapies in Medicine. 2004; 12(1):28-37. [DOI:10.1016/j. ctim.2003.12.003] [PMID]

[36] Johannessen B. Nurses experience of aromatherapy use with dementia patients experiencing disturbed sleep patterns. An action research project. Complementary Therapies in Clinical Practice. 2013; 19(4):209-13. [DOI:10.1016/j.ctcp.2013.01.003] [PMID]

[37] Ghods AA, SotodehasI N, Ghorbani R, Valian, M. [The effects of lavendula aroma on the accuracy of intensive care nurses (Persian)]. Journal of Babol University of Medical Sciences. 2013; 15(5):45-51. http:// jbums.org/article-1-4519-fa.pdf

[38] Peana AT, De Montis MG, Nieddu E, Spano MT, Paolo SD, Pippia P. Profile of spinal and supra-spinal antinociception of (-)-linalool. European Journal of Pharmacology. 2004; 485(1-3):165-74. [DOI:10.1016/j. ejphar.2003.11.066] [PMID]

[39] Abuhamdah S, Chazot PL. Lemon Balm and Lavender herbal essential oils: Old and new ways to treat emotional disorders? Current Anaesthesia \& Critical Care. 2008; 19(4):221-6. [DOI:10.1016/j.cacc.2008.05.005]

[40] Koto R, Imamura M, Watanabe C, Obayashi S, Shiraishi M, Sasaki Y. Linalyl acetate as a major ingredient of lavender essential oil relaxes the rabbit vascular smooth muscle through dephosphorylation of myosin light chain. Journal of Cardiovascular Pharmacology. 2006; 48(1):850-6. [DOI:10.1097/01.fjc.0000238589.00365.42] [PMID] 